\title{
ANÁLISE DOS REGISTROS DE INTOXICAÇÃO POR AGROTÓXICOS EM GOIÁS, NO PERÍODO DE 2001 A 2004.
}

\section{ANAL YSIS OF THE REGISTRATIONS OF INTOXICATION FOR PESTICIDES IN GOIAS, IN THE PERIOD FROM 2001 TO 2004.}

\author{
Adeline M. Silva ${ }^{1,2}$; Sueli M. de F. Alves ${ }^{3}$ \\ ${ }^{1}$ Bióloga, Especialista no Ensino de Biologia. Núcleo Regional de Polícia Técnico-Científica - Secretária de \\ Segurança Pública. Av.: Mato Verde, s/n. Setor Jibran El Hadj. 75000-000. Anápolis-GO. \\ ${ }^{2}$ Este artigo é parte da monografia do curso de Especialização no Ensino de Biologia - Unidade Universitária de \\ Ciências Exatas e Tecnológicas, Universidade Estadual de Goiás - UEG. \\ ${ }^{3}$ Doutora. Unidade Universitária de Ciências Exatas e Tecnológicas, Universidade Estadual de Goiás - UEG. \\ Caixa Postal 459 - CEP 75110-390. Anápolis-GO.
}

*Autor para correspondência e-mail: adelinemiranda@bol.com.br

Recebido em 20/08/2007 - Aceito em 09/11/2007

RESUMO: A larga utilização de agrotóxicos no controle de pragas promoveu um aumento dos problemas relacionados com as intoxicações com tais produtos. Esse quadro levou a promulgação de diversas leis e decretos, a fim de regulamentar, fiscalizar e oferecer serviços de atendimento aos intoxicados, além de quantificar e qualificar os acidentes. O Sistema Nacional de Informações Toxicológicas (Sinitox) divulga o número de casos registrados de intoxicações por diversos agentes tóxicos junto aos Centros de Informações Toxicológicas (CIT). Os registros são feitos por meio de fichas de notificação e atendimento, que são arquivadas em cada CIT. As fichas arquivadas nos CIT constituem uma fonte rica de informações. O objetivo deste trabalho foi analisar as fichas de notificação e atendimento, dos anos de 2001 a 2004, referentes à intoxicação por agrotóxicos, disponibilizadas pelo CIT - Goiás. Após a organização dos dados, procedeu-se a análise descritiva das informações. Verificou-se que a principal circunstância de ocorrência de intoxicação corresponde às notificações de tentativa de suicídio, as quais apresentam uma tendência de aumento nos meses de outubro a janeiro. O maior número de registros são provenientes do município de Goiânia.

PALAVRAS-CHAVES: Agrotóxicos, notificações de intoxicações e tentativa de suicídio.

ABSTRACT: The wide pesticides use in the control of plagues promoted an increase of the problems related with the intoxications with such products. That picture took the promulgated of several laws and ordinances, in order to regulate, to inspect and to offer attendance services to those intoxicated, besides to quantify and to qualify the accidents. The National System of Toxicological Information (Sinitox) it discloses the number of registered cases of intoxications for several toxicant agents, close to the Centers of Toxicological Information (CIT). The registrations are made by means of notification records and attendance, which are filed in each CIT. The records filed in CIT constitute a rich source of information. The objective of this work went analyze the notification records and attendance, of the referring years from 2001 to 2004, to the intoxication for pesticides, disposability for CIT - Goias. After the organization and tabulation of the information, the descriptive and exploratory analysis of the data was proceeded. It was verified that the main circumstance of intoxication occurrence corresponds to the notifications of suicide attempt, which present an increase tendency in the months of October to January. The largest number of registrations is coming of the municipal district of Goiânia.

KEYWORDS: Pesticides, notifications of intoxications and suicide attempt 


\section{INTRODUÇÃO}

Substâncias químicas com fins de controle de pragas e doenças têm registros entre os escritos gregos e romanos de mais de 3.000 anos. No Brasil, a introdução dos agrotóxicos foi feita de forma desorganizada, acompanhada de pacotes tecnológicos que introduzia a mecanização em larga escala, associada a outros fatores de produção. Neste quadro, o enfoque básico é o aumento da produtividade sem considerar riscos à saúde ou ao meio ambiente. Ao longo do tempo foram observados diversos casos de contaminação ambiental e de problemas de saúde pública, intoxicações de trabalhadores rurais e resíduos em alimentos. Esses fatores desencadearam o reconhecimento dos riscos decorrentes do uso abusivo dos agrotóxicos (OPAS, 1997; ALVES FILHO, 2002; SOBREIRA, 2003; LUNA et al., 2004; NOVATO-SILVA et al., 2004; NUNES \& TAJARA, 1998; CALDAS \& SOUZA, 2000; MOREIRA et al., 2002).

O impacto de uso de agrotóxicos sobre a saúde humana é um problema que tem merecido atenção da comunidade científica em todo o mundo, sobretudo nos países em desenvolvimento, onde se observa o maior número de mortes decorrentes da exposição humana a esses agentes. O aumento dos problemas relacionados aos agrotóxicos de uso agrícola, principalmente, levou a criação da Lei dos Agrotóxicos em 1989, a partir de então - Ministério da Saúde começou a implantar junto ao sistema de controle de informações toxicológicas, a investigação dos acidentes com agrotóxicos, utilizando fichas de notificação e atendimento. Esse monitoramento tem como objetivo primordial expor a situação das intoxicações por agrotóxicos e delimitar campos de atuação, a fim de reduzir o número de acidentes (ALVES FILHO, 2002; MOREIRA et al., 2002; GARCIA et al. 2005; ARAÚJO et al., 2000).

Os Centros de Informação Toxicológicas tem como atribuição, além da coleta e processamento de dados, analisar e interpretar as informações geradas. Essa análise e interpretação são realizadas para que possam recomendar e aplicar medidas de controle apropriadas a cada região, avaliando ainda se as medidas adotadas estão sendo efetivas, eficientes e divulgar as informações pertinentes. Seguindo essas diretrizes, partes dos dados coletados nos Centros de Informações Toxicológicas são divulgados pelo Sistema Nacional de Informações Toxicológicas (Sinitox), que apresenta o quadro geral dos acidentes notificados em todo o país. Todo esse processo encontra-se comprometido pela sub-notificação que se configura pelos casos que os CIT's não têm acesso, seja por questões de localização dos CIT's, desconhecimento da população, entre outros. Porém, para que todo esse processo possa ser gerido da melhor forma possível, os dados obtidos devem ser de qualidade, fidedignos, em grande quantidade, para que a informação possa indicar a magnitude do problema. No entanto, enquanto não há dados em quantidade e qualidade ideais, o estudo dos dados disponíveis até o momento se faz necessário (OPAS, 1997; BRASIL, 2003; POLASTRO, 2005).

No presente objetivou-se estabelecer o perfil geral das intoxicações por agrotóxicos de uso agrícola notificadas ao Centro de Informações Toxicológicas de Goiás, nos anos de 2001 a 2004.

\section{MATERIAIS E MÉTODOS}

O trabalho foi realizado durante o ano de 2005, junto ao Centro de Informações Toxicológicas de Goiás (CIT - GO), situado a Avenida Anhanguera, 5195 - Setor Coimbra, Goiânia (GO).

Os dados utilizados neste estudo são provenientes tanto do Sistema Nacional de Informações Tóxico Farmacológicas (Sinitox), quanto do Centro de Informações Toxicológicas de Goiás (CIT). O Sinitox, com sede na Fundação Oswaldo Cruz (Fiocruz), foi criado pelo Ministério da Saúde em 1980 e disponibiliza informações sobre os Centros de Controle de Intoxicações espalhados pelo país e atualmente armazena dados de 1985 até 2004.

Os dados disponibilizados na página do Sinitox (www.fiocruz.br/sinitox/) do Sinitox representam uma fonte de estudos sobre as notificações de intoxicação por agrotóxicos no Brasil. Os dados das Fichas de Notificação e Atendimento, registradas no CIT-GO, constituem uma fonte rica de informação por representarem informações complementares não divulgadas oficialmente. Nesse caso as informações podem ser reelaboradas de acordo com o objetivo da pesquisa (SEVERINO, 2000; CERVO \& BERVIAN, 2002; Gil, 2002).

As Fichas de Notificação e Atendimento, das quais os dados foram retirados, estão arquivadas no CIT de Goiás e estão à disposição para consulta mediante identificação junto à Biblioteca do CIT. Para o presente estudo foi selecionado o período compreendido de 2001 a 2004.

As informações contidas nessas fichas obedecem a uma padronização, para todo o estado, com a maior parte dos campos com o preenchimento por meio de opções predeterminadas. A ficha é composta de quarenta e quatro itens e dividem-se em dados gerais, dados do intoxicado, dados do evento, dados do agente tóxico, dados complementares, manifestações clínicas e evolução.

a - Dados gerais: compreendem os dados do solicitante como nome, a unidade de saúde que porventura ele representa, assim como o município e o telefone de contato.

b - Dados do intoxicado: compõem-se do nome, filiação, data de nascimento, idade, sexo, peso, ocupação e localidade de residência do intoxicado. 
c - Dados do evento: informam o nome do local onde ocorreu o evento, município, zona, local, evento, via de exposição, exposição (via de intoxicação), duração e tempo de exposição ao agente tóxico, circunstância do incidente e no caso de acidente de trabalho, a possível Comunicação de Acidentes de Trabalho.

d - Dados do agente tóxico: permitem uma descrição pormenorizada, já que há campos específicos para o nome comercial ou popular, o princípio ativo ou nome científico e a dose do agente tóxico a qual o intoxicado foi exposto.

e - Dados complementares: expõem o quadro clínico do intoxicado, incluem a confirmação laboratorial, se houve manifestações clínicas, a avaliação do grau de intoxicação do paciente.

f - Manifestações clínicas: neste quadro descrevem-se os sintomas e outras informações clínicas como a pressão arterial, freqüência cardíaca, temperatura do paciente no momento do atendimento entre outros.

g - Evolução: pode ser descrita com detalhes ou apenas com a definição do caso (cura, óbito, cura não confirmada etc).

Há um campo específico para a classificação do grupo do agente tóxico, que varia desde medicamentos, bebidas, drogas de abuso, animais peçonhentos etc. Neste campo encontra-se a divisão usada pelo Sinitox, que agrupa os tipos de agrotóxicos em agrotóxicos "de uso agrícola" (produtos direcionados para uso no campo) e de uso doméstico.

A transcrição de dados foi realizada após uma análise prévia das fichas. Selecionou-se o período de 2001 a 2004, para coleta, pelo fato de haver uma padronização tanto no modelo quanto no preenchimento da fichas.

Após a transcrição das informações, os dados foram tabulados com o objetivo de realizar a análise estatística. Nessa etapa procedeu-se a análise descritiva dos dados, por meio da construção de tabelas de distribuição de freqüência simples e dupla entrada e cálculo de porcentagem (COSTA NETO, 2002; COLLEGARIJACQUES, 2004; ARANGO, 2005).

\section{RESULTADOS}

Segundo os dados divulgados pelo Sinitox, o Centro-Oeste ocupa o $4^{\circ}$ lugar na classificação nacional, em relação aos registros de intoxicações por agrotóxicos "de uso agrícola", registradas no ano de 2004. O número de registros do Centro-Oeste corresponde a $7,15 \% ; 7,43 \%$ e $7,18 \%$ do total de notificações de intoxicação humana registrados nos anos de 2001, 2002 e 2003, respectivamente (SINITOX, 2007). O estado de Goiás, no mesmo período, foi responsável por $4,57 \% ; 4,75 \%$ e $4,49 \%$ dos registros.

A partir dos dados obtidos das fichas de notificações registradas no Centro de Informações Toxicológicas de Goiás, apuraram-se 245, 259, 257 e 299 notificações de eventos relacionados com agrotóxicos "de uso agrícola", respectivamente, nos anos de 2001, 2002, 2003 e 2004.

Na Figura 1 se encontra a distribuição das notificações ao longo do ano e observa-se que nos meses de novembro, dezembro e janeiro ocorreram os maiores números de registro de intoxicações. Nestes três meses ocorreram 36,29\% dos registros de intoxicações no ano de 2002, 40,80\% em 2003 e 33,07\% em 2004. 


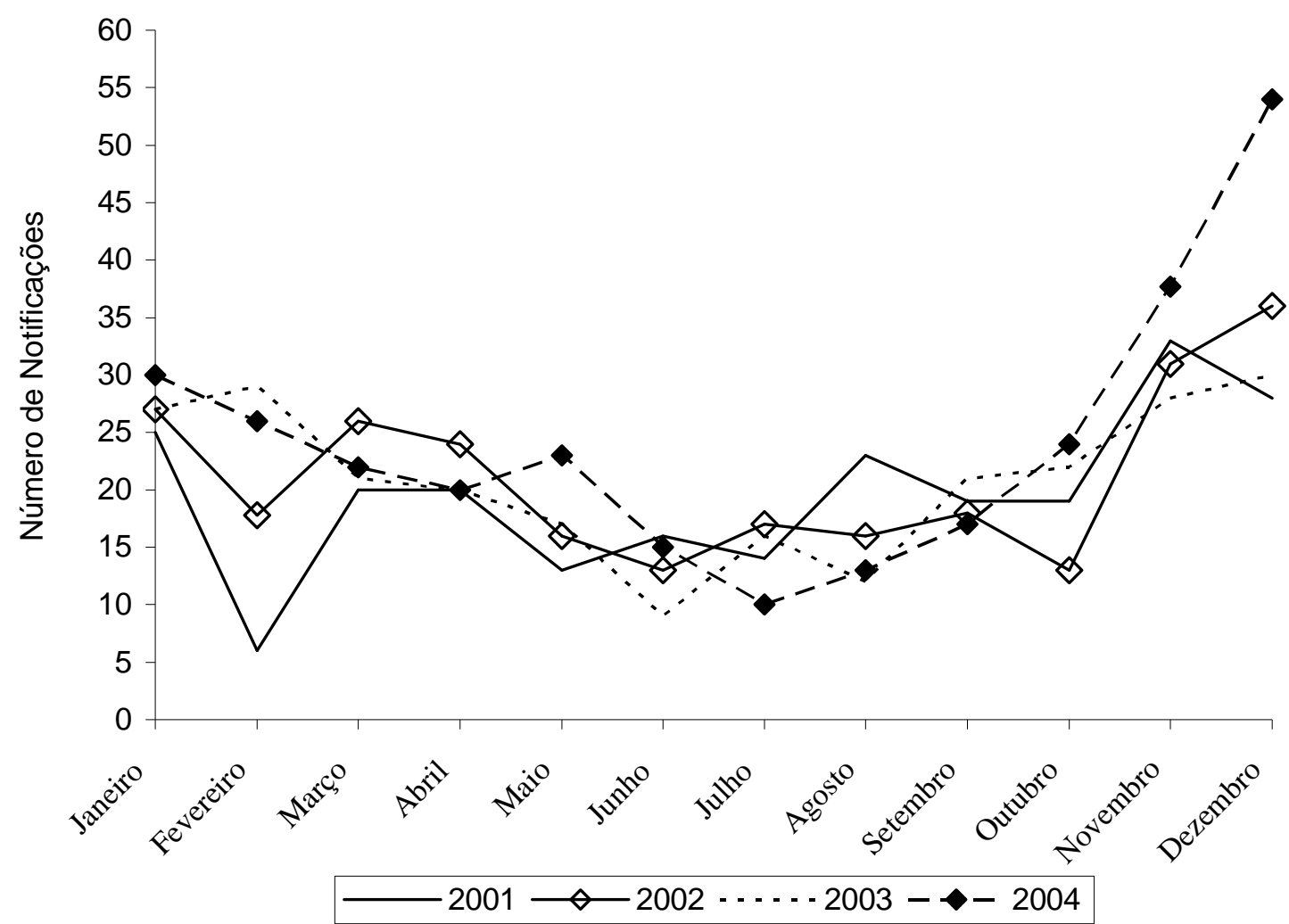

Figura 1. Distribuição dos registros no CIT, em função dos meses das notificações por agrotóxicos "de uso agrícola", de 2001 a 2004 (Fichas de Notificação e Atendimento do Centro de Informações Tóxico-Farmacológicas de Goiás - CIT-GO).

A maior parte das intoxicações com agrotóxicos "de uso agrícola" notificados, ocorreram com pessoas da faixa etária de 20 a 50 anos.

Como principais locais de ocorrência dos eventos com agrotóxicos "de uso agrícola", tem-se a residência e o ambiente de trabalho que correspondem, respectivamente, a mais de $35 \%$ e $11 \%$ das notificações em todos os anos pesquisados.

A Tabela 1, agrupa as notificações por local, circunstância durante os anos e observa-se que o maior número de registros, nos quatro anos, são os casos de tentativa de suicídio, ocorrido em residências.

Considerando apenas as circunstâncias em que ocorrem os eventos com agrotóxicos "de uso agrícola", o maior número de notificações ocorreram com tentativas de suicídio, que correspondem a $42,45 \%$ dos casos no ano de 2001 , 35,91\% em 2002, 49,81\% em 2003 e 36,12\% em 2004 do total de notificações.

Tabela 1. Local de maior ocorrência relacionadas às circunstâncias das notificações de intoxicação por agrotóxicos "de uso agrícola" no CIT de Goiás, 2001-2004

\begin{tabular}{|c|c|c|c|c|c|c|c|}
\hline Ano & Circunstância & $\begin{array}{c}\text { Ambiente } \\
\text { de } \\
\text { Trabalho }\end{array}$ & $\begin{array}{l}\text { Ambiente } \\
\text { Externo }\end{array}$ & Residência & $\begin{array}{c}\text { Residência / } \\
\text { Ambiente de } \\
\text { Trabalho }\end{array}$ & Outro & Não Informado \\
\hline & Tentativa de suicídio & 2 & 2 & 75 & -- & $2 ;$ & 23 \\
\hline \multirow[t]{4}{*}{2001} & Acidente de Trabalho & 36 & -- & 5 & -- &.-- & 5 \\
\hline & Acidente Individual & 17 & 2 & 36 & 1 & -- & 9 \\
\hline & Outros & 1 & -- & 21 & -- & $--\cdot$ & 8 \\
\hline & Tentativa de suicídio & 3 & -- & 58 & -- & $\cdots$ & 32 \\
\hline \multirow[t]{4}{*}{2002} & Acidente de Trabalho & 33 & -- & 1 & 1 &.-- & 17 \\
\hline & Acidente Individual & 19 & 1 & 28 & -- & 16 & 16 \\
\hline & Outros & 6 & -- & 10 & -- & 10. & 23 \\
\hline & Tentativa de suicídio & 4 & 2 & $71^{-}$ & -- & $\cdots$ & 51 \\
\hline \multirow[t]{4}{*}{2003} & Acidente de Trabalho & -- & -- & -- & -- & -- & 12 \\
\hline & Acidente Individual & 8 & -- & 22 & -- &..- & 19 \\
\hline & Outros & 23 & -- & 8 & -- & $--\cdot$ & 37 \\
\hline & Tentativa de suicídio & -- & -- & 60 & -- & $\cdots$ & 48 \\
\hline
\end{tabular}




$\begin{array}{lrrrrrr}2004 \text { Acidente de Trabalho } & 27 & 1 & 5 & -- & --. & 60 \\ \text { Acidente Individual } & 7 & -- & 26 & -- & 1 . & 27 \\ \text { Outros } & 1 & -- & 14 & -- & --. & 22\end{array}$

Fonte: Fichas de Notificação e Atendimento do Centro de Informações Tóxico-Farmacológicas de Goiás CIT-GO.

Quanto às notificações por sexo, zona e a circunstância observa-se que o maior número de registros, nos quatro anos, foram os casos de tentativa de suicídio, ocorridos na zona urbana independentemente do sexo (Tabela 2). Por outro lado, na zona rural, a maior parte das notificações foram com indivíduos do sexo masculino acometidos de acidentes de trabalho. MENDONÇA \& MARINHO (2005), observaram que o maior número de registros de intoxicações por agrotóxicos de uso agrícola $(64,51 \%)$, no meio rural são em indivíduos do sexo masculino, bem como uma expressiva atuação deste agente em acidentes ocupacionais $(28,17 \%)$. No presente estudo, o maior número de notificações com agrotóxicos "de uso agrícola", ocorreu na zona urbana (Tabela 3). Em relação aos municípios verifica-se que o maior número de casos registrados de intoxicações por agrotóxicos de uso agrícola no estado de Goiás, foi em Goiânia e cidades circunvizinhas.

Tabela 2. Notificações intoxicações por agrotóxicos agrupadas por zona, sexo e circunstância, registradas junto ao Centro de Informações Tóxico-Farmacológicas de Goiás (CIT-GO), 2001-2004

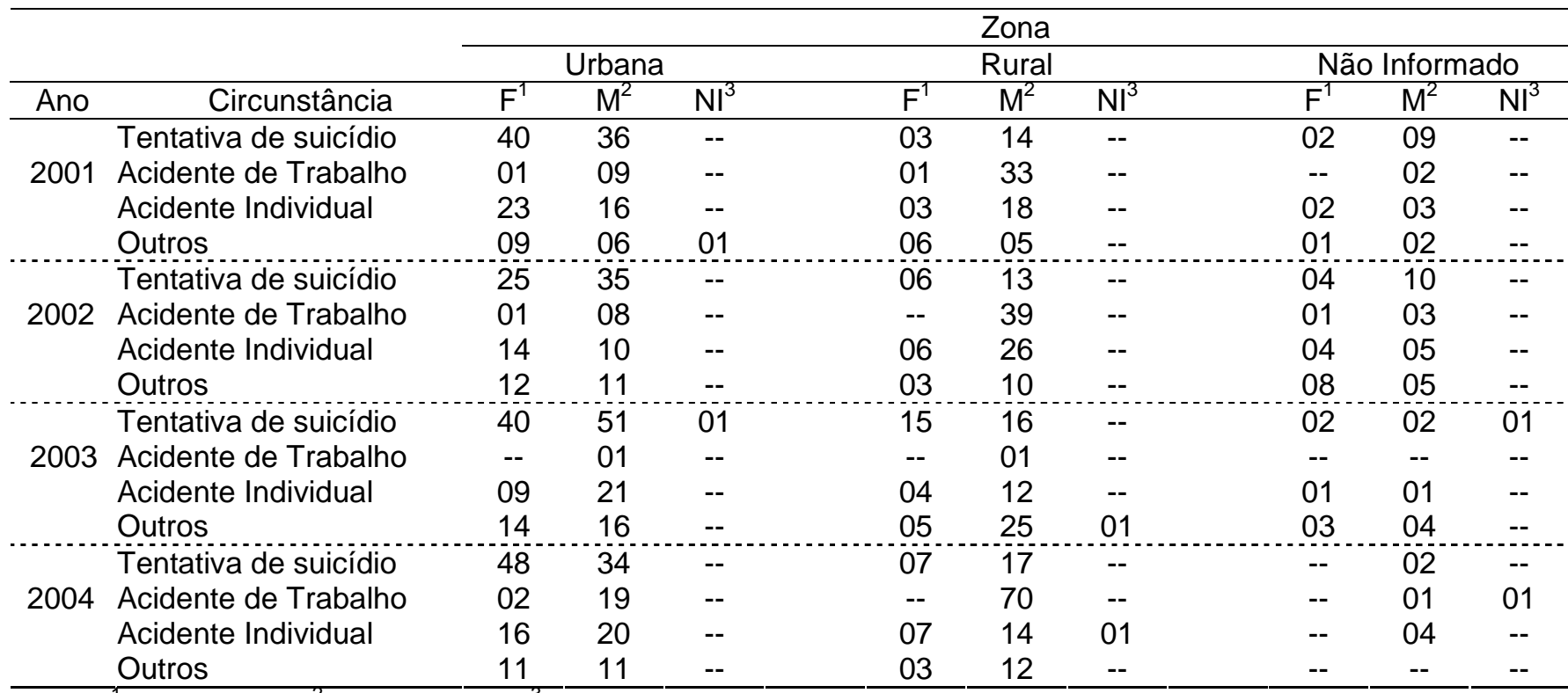

$\mathrm{F}^{1}$ : Feminino; $\mathrm{M}^{2}$ : Masculino; $\mathrm{NI}^{3}$ : Não Informado.

Fonte: Fichas de Notificação e Atendimento do Centro de Informações Tóxico-Farmacológicas de Goiás - CIT-GO.

Quanto aos casos de tentativa de suicídio, registraram-se 104, 93, 128 e 108 casos de tentativa de suicídio, nos anos de 2001, 2002, 2003 e 2004, respectivamente. Destes casos a evolução para a cura corresponde a $86,5 \%$ dos casos em 2001, 69,9\% em 2002, 74,2\% em 2003 e 74\% em 2004. Se compararmos com o total geral de notificações por agrotóxicos "de uso agrícola", verifica-se que a evolução para cura compreende $86,12 \%$, $74,90 \%, 81,71 \%$ e $83,28 \%$ dos casos registrados nos anos de 2001, 2002, 2003 e 2004, respectivamente. 
Tabela 3. Municípios e zona de ocorrência com maiores registros de intoxicações por agrotóxicos junto ao CIT de Goiás, 2001-2004

\begin{tabular}{|c|c|c|c|c|c|c|c|c|c|c|c|c|c|c|c|}
\hline Ano & Zona & 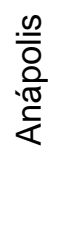 & 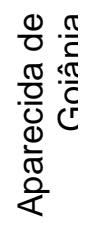 & $\begin{array}{l}\frac{\pi}{0} \\
\stackrel{\frac{\pi}{\infty}}{\Phi} \\
\frac{\pi}{0}\end{array}$ & 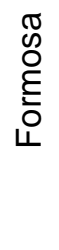 & $\begin{array}{l}\frac{\pi}{0} \\
\stackrel{\mathscr{d}}{\leftarrow} \\
\stackrel{\frac{\pi}{0}}{0} \\
\mathbb{0}\end{array}$ & 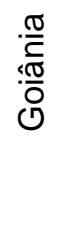 & 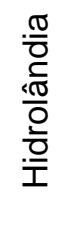 & 需 & 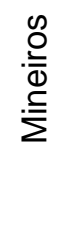 & 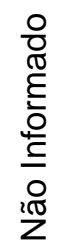 & 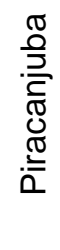 & $\begin{array}{l}\frac{0}{0} \\
\frac{0}{0} \\
\frac{0}{\pi} \\
\frac{0}{\alpha}\end{array}$ & 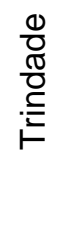 & 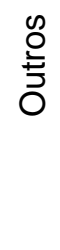 \\
\hline \multirow{3}{*}{2001} & Urbana & 07 & 09 & 02 & 02 & 01 & 49 & 03 & 12 & -- & 25 & 01 & 02 & 02 & 26 \\
\hline & Rural & 04 & 01 & 01 & -- & 01 & 02 & 04 & 17 & 01 & 19 & -- & 01 & -- & 35 \\
\hline & Não Informada & 01 & $\therefore$ & 01 & $\therefore$ & - & 07 & -- & $\therefore$ & $\therefore$ & 07 & $\therefore$ & - & 01 & 04 \\
\hline \multirow{3}{*}{2002} & Ürbana & 08 & 07 & -- & 02 & 01 & 30 & 02 & 16 & 01 & 10 & -- & 02 & 02 & 35 \\
\hline & Rural & -- & 01 & 06 & 01 & 02 & 02 & 01 & 25 & 02 & 04 & -- & 07 & 01 & 51 \\
\hline & Não Informada & 02 & 01 & $\therefore$ & 02 & -- & 03 & $\therefore-$ & $\therefore$ & 01 & 13 & 01 & 01 & $\therefore-$ & 16 \\
\hline \multirow{3}{*}{2003} & Ürbana & 11 & 11 & 01 & 05 & -- & 29 & -- & 14 & 03 & 29 & 02 & 14 & 06 & 28 \\
\hline & Rural & 04 & -- & 05 & 07 & 01 & 07 & 01 & 20 & 01 & 14 & 01 & 06 & 01 & 22 \\
\hline & Não Informada & 01 & 01 & - & 01 & -- & $\therefore$ & -- & $\therefore$ & -- & 07 & $\therefore-$ & 01 & 01 & 02 \\
\hline \multirow{3}{*}{2004} & Ürbana & -- & 06 & 02 & -- & 04 & 42 & - & 15 & 01 & 24 & 01 & 11 & 02 & 53 \\
\hline & Rural & -- & -- & 04 & -- & 01 & 04 & 02 & 24 & 01 & 09 & 01 & 07 & 01 & 76 \\
\hline & Não Informada & -- & -- & -- & -- & -- & -- & -- & -- & -- & 04 & 01 & -- & -- & 02 \\
\hline
\end{tabular}

Fonte: Fichas de Notificação e Atendimento do Centro de Informações Tóxico-Farmacológicas de Goiás - CIT-GO.

\section{DISCUSSÃO}

Estima-se que os casos registrados de intoxicação representam somente $2 \%$ do total de casos ocorridos anualmente. Vale ressaltar, que as estatísticas divulgadas pelo Sistema Nacional de Informações Tóxicofarmacológicas (Sinitox) não contemplam a totalidade dos casos de intoxicação humana por agrotóxicos ocorridos no Brasil, pois se referem apenas aos casos registrados de intoxicações agudas. Assim, estes dados referem-se somente a casos de intoxicações que foram notificados nos centros de informações toxicológicas(GARCIA \& ALVES FILHO, 2005).

As agências internacionais de saúde, como a OMS, consideram que em países como o Brasil, no qual existem graves problemas de estrutura da saúde pública, ocorre um sub-registro de casos de intoxicações por agentes químicos em geral (TRAPÉ, 2003).

Esses números podem não representam a real dimensão do problema, uma vez que se deve considerar que as intoxicações agudas são apenas uma parte dos danos causados à saúde humana. Quando se considera os casos de intoxicações sub-crônicas e crônicas, que não serão abordados neste trabalho, pode-se verificar que as dimensões do problema assumem proporções maiores. Neste contexto, os efeitos indiretos da presença dos agrotóxicos no ambiente podem ser tão importantes quanto os efeitos diretos da toxicidade. Esses efeitos crônicos da contaminação podem interferir em fatores como: expectativa de vida, crescimento, fisiologia, comportamento e reprodução dos seres vivos expostos. Como exemplos mais comuns de efeitos crônicos de exposição a agrotóxicos têm-se as dermatoses, o câncer e seqüelas neurocomportamentais (LARINI \& CECCHINI, 1987; CALDAS \& SOUZA, 2000; ALVES FILHO, 2002;).

Os dados apresentados pelos Centros de Informações Toxicológicas não refletem a real dimensão do problema com agrotóxicos "de uso agrícola", uma vez que advêm de Centro de Controle de Intoxicações situados em centros urbanos, inexistentes em várias regiões produtoras importantes, ou de difícil acesso para as populações rurais, além da falta de informação acerca da existência dos Centros de Informações Toxicológicas (SCHVARTSMAN, 1991; MOREIRA et al., 2002; TRAPÉ, 2003).

Tais fatores podem estar relacionados tanto ao elevado número de notificações registradas na zona urbana, como o fato de Goiânia liderar a classificação geral das cidades com maior índice de notificações.

O elevado número de notificações cuja circunstância é a tentativa de suicídio, podem estar relacionadas ao amplo acesso aos agrotóxicos "de uso agrícola" sem o controle necessário, assim como a disseminação de pragas que favorecem o incremento no consumo destes produtos na zona urbana (ALVES FILHO, 2002; TRAPÉ, 2003).

Em estudo semelhante realizado no estado do Paraná, verificou-se que o maior número de notificações por agrotóxicos, entre o período de 1993 a 2000, foi proveniente de acidentes profissionais, seguido dos casos de tentativa de suicídio (POLASTRO, 2005).

A faixa etária de 21 a 40 anos concentra mais de $48 \%$ dos registros de tentativa de suicídio, durante todos os anos pesquisados. Sendo que a faixa de 21 a 30 anos apresentou mais casos durante os anos de 2001, 2002 e 
2003. RIOS et al. (2005), observaram que nas notificações por medicamentos, cuja circunstância era a tentativa de suicídio, $68,51 \%$ dos casos se concentravam na faixa etária de 10 a 30 anos, sendo que a faixa de 21 a 30 anos representou $35 \%$ dos registros.

A maior parte das notificações registradas no CIT-GO tiveram como evolução a cura, que nestes casos corresponde, basicamente, a eliminação do quadro clínico sintomático apresentado pelo paciente no momento do atendimento, sem a realização de exames comprobatórios ou acompanhamento posterior, que poderiam evitar um novo quadro sintomático ou uma evolução para uma doença crônica.

Com base nos dados encontrados nas fichas de notificação e atendimento observa-se que a maior parte dos diagnósticos baseia-se na história, exame físico ou alterações patológicas e não na confirmação laboratorial, o que ajudaria a delimitar um tratamento mais específico para intoxicação, conforme o agente tóxico.

Faz-se necessário ressaltar que as informações apresentadas nas fichas muitas vezes não são preenchidas corretamente, o que prejudica a qualidade das informações. Este fator se deve ao não preenchimento, preenchimento incorreto ou incompleto dos campos da ficha. Esses aspectos refletem exatamente a situação das notificações registradas pelo CIT de Goiás, fato que foi observado durante toda a fase de coleta de dados, o que dificulta a determinação do perfil das notificações por agrotóxicos "de uso agrícola" em Goiás, embora se possa observar uma melhora relativa das informações no decorrer dos anos pesquisados. Esta mesma característica também foi verificada por POLASTRO (2005), no estudo dos casos de intoxicações por agrotóxicos no estado do Paraná.

\section{AGRADECIMENTOS}

Ao Centro de Informações Tóxico-Farmacológicas do Estado de Goiás (CIT-GO) pela disponibilização das Fichas de Notificação e Atendimento de Intoxicação em Goiás.

\section{CONCLUSÃO}

O estudo dos registros de intoxicação por agrotóxicos de uso agrícola no estado de Goiás caracterizou a circunstância de tentativa de suicídio como a principal causa das intoxicações registradas no CIT-GO. Este resultado se contrapõe ao que era esperado no início das pesquisas, já que em se tratando de um produto voltado para a utilização com fins de controle de pragas agrícolas, a intoxicação devido ao acidente de trabalho parecia ser a causa mais provável. Contudo ao analisar estes dados, deve-se considerar que há um alto índice de subnotificação de casos de intoxicação por agentes químicos.

\section{REFERÊNCIAS BIBLIGRÁFICAS}

ALVES FILHO, J. P. Uso de Agrotóxicos no Brasil - Controle Social e Interesses Corporativos. 1a ed. São Paulo: Annablume; Fapesp, 2002.

ARANGO, H. G. Bioestatística: Teórica e Computacional. 2ª ed. Rio de Janeiro: Guanabara Koogan, 2005.

ARAÚJO, A. C. P.; NOGUEIRA, D. P. \& AUGUSTO, L. G. S. Impacto dos Praguicidas na saúde: estudo da cultura de tomate. Revista de Saúde Pública. São Paulo, v. 34, no. 3, 2000.

BRASIL. Ministério da Saúde. Agencia Nacional de Vigilância Sanitária (ANVISA). Informativo de Procedimentos para Avaliação Toxicológica de Agrotóxicos seus Componentes e Afins. Brasília, 2003. Disponível em: http://www.anvisa.gov.br/toxicologia/ estrutura/index.htm. Acesso em: 24 jun. 2005.

CALDAS, E. D. \& SOUZA, L. C. K. R. Avaliação de Risco Crônico da Ingestão de Resíduos de Pesticidas na Dieta Brasileira. Revista de Saúde Pública. São Paulo, v. 34, №. 5, out, 2000.

CERVO, A. L. \& BERVIAN, P. A. Metodologia Científica. 5ª ed. São Paulo, Prentice Hall, 2002.

COLLEGARI-JACQUES, S. M. Bioestatística: Princípios e Aplicações. Porto Alegre: Artmed, 2004.

COSTA NETO, P. L. O. Estatística. São Paulo: Editora Edgard Blucher, 2002.

GARCIA, E. G. \& ALVES FILHO, J. P. Aspectos de Prevenção e Controle de Acidentes no Trabalho com Agrotóxicos. São Paulo: Fundacentro, 2005.

GARCIA, E. G.; BUSSACOS, M. A. \& FISCHER, F. M. Impacto da Legislação no Registro de Agrotóxicos de Maior Toxicidade no Brasil. Revista de Saúde Pública. São Paulo, v. 39, №. 5, out, 2005. 
GIL, A. C. Como Elaborar Projetos de Pesquisa. 4를 ed. São Paulo: Editora Atlas, 2002.

LARINI, L. \& CECCHINI, R. A Intoxicação Como Fenômeno Biológico. In: LARINI, Lourival. Toxicologia. 1ae ed. São Paulo: Editora Manole, 1987. p. 01-41.

LUNA, A. J.; SALES, L. T. \& SILVA, R. F. Agrotóxicos: Responsabilidade de Todos (Uma abordagem da questão dentro do paradigma do desenvolvimento sustentável). 2004. Disponível em: shttp: saudeetrabalho.com.br/download/agrotoxicos-responsabilidade.doc $>$. Acesso em: 24 nov. 2005.

MENDONÇA, R. T. \& MARINHO, J. L. Discussão Sobre Intoxicações Por Medicamentos e Agrotóxicos no Brasil de 1999 a 2002. Revista Eletrônica de Farmácia. v. 2, №. 02, 2005. p. 45-63.

MOREIRA, J. et al. Avaliação Integrada do Impacto do Uso de Agrotóxicos Sobre a Saúde Humana em uma Comunidade Agrícola de Nova Friburgo, RJ. Ciência e Saúde Coletiva. v. 7, no․ 02, 2002. p. 299-311.

NOVATO-SILVA, E.; SILVA, J. M. ; SOUZA, R. A.; RODRIGUES, F. A. L. \& SILVA, G. M. E. Educação Para a Saúde: o Conhecimento como Ferramenta de Redução dos Riscos da Exposição Ocupacional à Agrotóxicos. In: Anais do 7º Encontro de Extensão da Universidade Federal de Minas Gerais. Belo Horizonte (MG), 2004.

NUNES, M. V. \& TAJARA, E. H. Efeitos Tardios dos Praguicidas Organoclorados no Homem. Revista de Saúde Pública. v. 32, ํo. 4, ago, 1998.

ORGANIZAÇÃO PAN-AMERICANA DE SAÚDE (OPAS). Manual de Vigilância da Saúde de Populações Expostas à Agrotóxicos. Brasília, 1997. Disponível em: http://www.opas.org.br/sistema/arquivos/livros2.pdf. Acesso em: 08 jul. 2005.

POLASTRO, D. Estudo dos casos de intoxicação ocasionadas pelo uso de agrotóxicos no estado do Paraná, durante o período de 1993 a 2000. Dissertação de Mestrado, Universidade de São Paulo, 2005.

RIOS, D. P.; BASTOS, F. M.; CUNHA, L. C. \& VALADARES, M. C. Tentativa de Suicídio Com o Uso de Medicamentos Registrados pelo CIT-GO nos Anos de 2003 e 2004. Revista Eletrônica de Farmácia. v. 2, №. 01, 2005. p. 6-14.

SCHVARTSMAN, S. Intoxicações Agudas. 4ํed. São Paulo: Editora Sarvier, 1991.

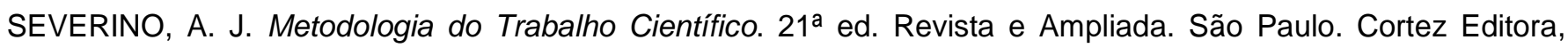
2000.

SOBREIRA, A. E. G. \& ADISSI, P. J. Agrotóxicos: Falsas Premissas e Debates. Ciência e Saúde Coletiva. v. 8, no 4, 2003.

TRAPÉ, A. Z. Efeitos Toxicológicos e Registros de Intoxicações por Agrotóxicos. Campinas, 2003. Disponível em: http://www.feagri.unicamp.br/tomates/pdfs/eftoxic.pdf Acesso em: abr. 2005. 